\title{
LA CONSTRUCCIÓN DE REALIDADES INSEGURAS. REFLEXIONES ACERCA DE LA VIOLENCIA EN CENTROAMÉRICA
}

\section{THE CONSTRUCTION OF UNSAFE REALITIES: REFLECTIONS ON VIOLENCE IN CENTRAL AMERICA}

\author{
Sebastian Huhn* \\ Anika Oettler** \\ Peter Peetz****
}

RESUMEN

En todos los países de Centroamérica se está llevando a cabo una lucha de definición, interpretación y clasificación entorno al campo temático de la violencia, la delincuencia y la inseguridad. Y aunque esta lucha tenga consecuencias políticas y sociales sumamente relevantes, no ha sido objeto de un análisis sistemático. Los autores argumentarán que no son los fenómenos de violencia en sí que desatan histerias de inseguridad o que provocan la implementación de políticas criminales represivas. Más bien, la percepción de y las reacciones a la inseguridad y la violencia se basan en discursos sociales acerca de estos fenómenos.

PALABRAS CLAVES: AMÉRICA CENTRAL * VIOLENCIA * DELINCUENCIA * INSEGURIDAD * ANÁLISIS DEL DISCURSO

\section{ABSTRACT}

In all Central American countries we witness a struggle to define, interpret and classify types of violence, delinquency, crime and insecurity. Although this struggle has highly relevant political and social implications, it has not been analyzed systematically. The authors show that moral panic and repressive criminal policies are not direct consequences of the violence phenomena per se. Rather, the perception of and the reactions to insecurity and violence are based on social discourses about these phenomena.

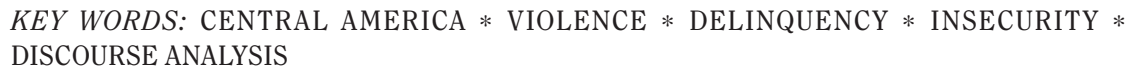

* Institute of Latin American Studies, Hamburgo, Alemania. /

huhn@giga-hamburg.de

*** Institute of Latin Amerian Studies, Hamburgo, Alemania. / peetz@giga-hamburg.de

** Institute of Latin American Studies, Hamburgo, Alemania. /

oettler@giga-hamburg.de 
INTRODUCCIÓN: LA IMPORTANCIA SOCIAL DE LA VIOLENCIA DELINCUENCIAL

En los años 80 y principios de los 90 Centroamérica captaba continuamente la atención mundial por el alto grado de violencia política de actores estatales y no-estatales. En Europa y Norteamérica, el interés de los medios de comunicación y de los analistas académicos por aquella región resultaba, en parte, del hecho de que los conflictos internos de estos países se interpretaban como manifestaciones del antagonismo global entre los dos bloques ideológicos y políticos de la guerra fría. Contrario a la situación de hoy, entre los intelectuales dentro y fuera de la región no existía en aquel entonces el amplio consenso de rechazar cualquier forma de violencia (Wieviorka, 2006).

Desde mediados de la década de los 90 , con el sucesivo fin de los conflictos armados en Nicaragua, El Salvador y Guatemala, el interés del mundo en Centroamérica disminuyó considerablemente, tanto en cuanto a la cobertura mediática como a la producción académica. Sin embargo, hoy en día, siguen siendo temas de violencia y seguridad las que más se discuten (aparte de temas de pobreza y desarrollo). Pero, aunque la violencia política no haya desaparecido completamente de la agenda, desde hace una década la preocupación central consiste en la violencia e inseguridad generada en el contexto de la delincuencia "común" o "cotidiana". Esa delincuencia se puede manifestar de muchas formas, por ejemplo en secuestros, asaltos, robos, asesinatos, en delincuencia relacionada con drogas, en violencia sexual y doméstica, y especialmente en la violencia juvenil.

Desde hace varios años prevalece en el istmo la percepción de que la ola de violencia política de la década de 1980 se disminuyó sólo para dar paso a una ola de violencia delincuencial. Para muchos, esta nueva ola representa una amenaza aún más grave que la anterior por dos razones: primero, porque en algunos países, sobre todo en El Salvador y Honduras, el grado de la violencia actual parece sobrepasar al que se registraba en los peores años de la época pasada; las estadísticas sobre "muertes violentas" parecen dejar poco lugar a dudas y encuentran un extensivo eco en los medios de comunicación $y$ en la esfera política. Segundo, porque hoy la violencia se percibe como más generalizada 0 "inevitable": se cree que la violencia política de antaño afectaba primordialmente a los que estaban "metidos en política" - lo que uno podía evitar - pero que la violencia criminal de hoy amenaza indiscriminadamente a todos los ciudadanos, independientemente de su orientación política, clase social, edad o descendencia étnica.

Consecuencia de esa percepción generalizada de la amenaza supuestamente omnipresente de la criminalidad son las medidas individuales y colectivas de protección y de "contraataque". Con relación a estas medidas, les conviene a gobiernos $y$ a otros actores hablar de "seguridad ciudadana". Esa expresión está connotada con un enfoque preventivo $y$, hasta cierto grado, liberal. Pone énfasis en la protección de los ciudadanos y contrasta con el concepto de la "seguridad nacional" que dominaba el discurso público en décadas pasadas y que enfocaba más en la protección y la defensa del Estado (véase también Zaffaroni, 1992). El cambio terminológico sugiere que los Estados ahora protegieran la integridad física, el patrimonio $y$ otros derechos individuales de todos los ciudadanos, aunque en muchos países las políticas de seguridad simplemente consistan en más represión.

Dependiendo de los recursos económicos disponibles de cada quien, el miedo a la violencia lleva a muchos ciudadanos a vivir en colonias cerradas, condominios vigilados (gated communities) o casas fortificadas; otros evitan frecuentar lugares identificados como peligrosos, sean mercados, determinadas calles o ciudades enteras. Aparte de las reacciones defensivas, crece la cantidad de personas que se arman o se organizan en comités de vigilancia. Paralelamente, se favorece a opciones políticas que prometen una lucha frontal, despiadada $y$ en muchos casos militarizada contra el crimen y "los criminales". Estas opciones políticas igual que las mencionadas tendencias desintegrativas, por última consecuencia, podrían poner en peligro unos procesos de democratización que en la mayoría de los países centroamericanos, ya de por si, no están avanzando con ímpetu. 
A pesar del predominio de tendencias desintegrativas y de opciones políticas poco favorables para la democracia, también existen espacios sociales en los que el ideario colectivo basado en principios solidarios fomenta prácticas colectivas tendientes a la integración social y la democratización. Sin embargo, parece que en Centroamérica, en general e independientemente de fronteras nacionales, prevalece un discurso represivo que corresponde con un estilo político autoritario. Esto implica una legitimación de políticas anti-delincuenciales de mano dura y conlleva a un debilitamiento de los principios del estado de derecho y de los derechos humanos. En Nicaragua, en cambio, la violencia juvenil $y$ las estrategias de mano dura no dominan los discursos políticos y mediales (véase Huhn/Oettler/Peetz, 2006).

Sin embargo, el tema de la seguridad personal se ha instalado como una de las principales preocupaciones de la ciudadanía, especialmente en los barrios pobres. El temor está ligado a la fuerte presencia del consumo y tráfico de drogas, a la ocupación del espacio público por "ladrones" $y$ violadores, $y$, finalmente, a la proliferación de pandillas juveniles.

Partiendo de estas observaciones se desarrollarán en este artículo unas hipótesis sobre la relación entre la percepción de la situación de violencia y seguridad en Centroamérica, el discurso público que genera esa percepción $(y$, al mismo tiempo, es fruto de ella) y los procesos sociales y políticos que se llevan a cabo en este contexto.

El término "discurso" se refiere a una "práctica de afirmaciones reguladas" (Foucault, 1997: 74) y se entiende como condición y consecuencia de prácticas colectivas. Los discursos construyen, transforman, estructuran y (mediante la repetición y la aceptación) consolidan prácticas colectivas.

\section{EL RECUENTO DE VÍCTIMAS: \\ LA PRECARIEDAD DE LA BASE EMPÍRICA}

La percepción generalizada de un aumento de violencia e inseguridad en Centroamérica se refleja claramente en la producción académica. Por ejemplo, en Guatemala, El Salvador,
Honduras y Nicaragua, varios equipos de investigación llevaron a cabo estudios acerca de la violencia juvenil, siendo la serie "Maras y pandillas en Centroamérica" (ERIC, et al. 2001, 2004a/b y Cruz, 2006) la más importante. Sin embargo, hay que subrayar que la investigación empírica se mueve en el ámbito de ciertas modas académicas que se generan a través de la búsqueda de temáticas novedosas o metodologías innovadoras (véase Huhn/Oettler, 2006). Además, el problema de la proliferación de las maras se convierte en un eje temático central de ONGs y organismos internacionales (por ejemplo, WOLA, USAID, BID). Hay que insistir, entonces, en el hecho de que la producción académica es una parte importante del discurso sobre la violencia en Centroamérica.

Esa producción académica se caracteriza - como en todo el mundo- por una paradoja: la base empírica es altamente cuestionable pero, sin embargo, se sigue recurriendo ampliamente a ella. Es más; en muchos casos datos empíricos constituyen el único fundamento para interpretar la realidad violenta de una sociedad. Pero hay aspectos claves de la temática que no se pueden debatir cabalmente dependiendo de esta base.

Para llegar a conclusiones sobre el nivel, las características, las causas y las consecuencias de la violencia en Centroamérica y Latinoamérica los estudios empíricos se basan casi exclusivamente en dos tipos de datos $y$, como dependen de estos datos, nunca pueden contener críticas fundamentales de ellos: estadísticas oficiales provenientes de diferentes agencias de gobierno (registros de policía, de órganos de justicia o de las instancias de salud pública) por un lado, $y$ datos de encuestas (encuestas de victimización o, en general, encuestas de opinión) por otro lado. En muchos casos se utilizan datos agregados de organizaciones internacionales (OMS, PNUD, etc.) que a su vez se componen o de datos propios de estas instituciones o bien de los mencionados datos de instituciones gubernamentales de los países. La pregunta ¿cuál de los dos tipos de datos (de estadísticas o de encuestas) es preferible? y ¿de qué manera se deben interpretar los datos? se discute en el marco de toda una controversia metodológica. Una corriente formula críticas generales 
(Muncie, 1996; Maguire, 2002; Schmidt, 2005), otra se empeña por el perfeccionamiento y la ampliación de las bases de datos y por aumentar la comparabilidad de las estadísticas internacionales (véase por ejemplo von Hofer, 2000; MacDonald, 2002).

Las limitaciones se acumulan cuando se intenta comparar datos de diferentes países. Muchos estudios comparativos elaborados por organizaciones internacionales aparentan reflejar tendencias recientes pero utilizan datos poco actuales $y$ poco comparables. El informe sobre violencia y salud de la OMS (WHO, 2002), por ejemplo, se basa en datos nacionales que fueron recolectados entre 1990 (Uruguay) y 1997 (México). Además, hay discrepancias en cuanto a la definición de la delincuencia y violencia. Los mecanismos de registro de actos criminales varían de país a país. Von Hofer (2000) ejemplifica los dos problemas con las estadísticas de abuso sexual en Suecia: primero, la definición sueca de lo que es violación y acoso sexual es bastante estricta en comparación con otros países. Segundo, los instrumentos de levantamiento de datos son relativamente sofisticados en aquella nación nórdica. Según von Hofer resulta de estos dos factores que el número de casos de abuso sexual en Suecia parece, en comparación con otros países europeos, bastante elevado. Para el contexto centroamericano pueden servir de ejemplo las políticas de "mano dura" en El Salvador y Honduras. Desde que se penalizó la pertenencia a una mara - por lo cual un tatuaje puede ser indicio suficiente $-y$ desde que en el trabajo policial se priorizó la persecución de delincuentes jóvenes, era previsible que en los registros de la policía, $y$ por lo tanto, en las estadísticas de delincuencia, el número de delitos perpetrados por jóvenes aumentara.

Otra causa de distorsiones entre las estadísticas de un país $y$ otro pueden ser diferencias en cuanto a la disposición de la víctima a denunciar un acto delictivo. En cada sociedad puede haber un consenso específico sobre lo que se denuncia, lo que se considera bagatela (aunque legalmente sea penalizado) y lo que es tabú. En el caso centroamericano habrá cierta homogeneidad en esa dimensión cultural por la historia compartida, la tradición católica, la cercanía geográfica y tantos otros aspectos que los cinco países tienen en común. Sin embargo, hay indicios de que también en esa región existen discrepancias en cuanto a lo que la sociedad define como tolerable. Podría ser, por ejemplo, que en una sociedad como la costarricense, donde la clase media con su correspondiente nivel educativo constituye un porcentaje de población (todavía) relativamente alto, la actitud para con jóvenes delincuentes tienda más hacia la comprensión y la ayuda que en sociedades marcadas por una fuerte desigualdad social y un nivel educativo más bajo, como en Honduras o El Salvador. Mucha importancia le corresponde, además, al hecho de que la presencia de la policía y de las instituciones judiciales diverge bastante en el istmo. Según Dennis Rodgers, en Nicaragua muchos delitos no entran a los registros policiales (y por lo tanto a las estadísticas criminales) por la total ausencia de la policía en el 21\% de los municipios del país. En otros municipios la presencia de la policía está limitada por causa de la reducción de personal que se efectuó en el marco de las sucesivas reformas policiales o recortes presupuestarios (Rodgers, 2004: 6). Las estadísticas criminales no pueden ser más que estadísticas de actividad policial o, respectivamente, de actividad judicial. Por lo tanto, el número registrado de actos violentos y delitos depende más del trabajo de las agencias estatales de seguridad y justicia, $y$ de su equipamiento con recursos humanos $y$ económicos, que del número real de crímenes que se cometen: “Un 'incremento del crimen' se puede deber a más crímenes reportados, en vez de más crímenes cometidos". (Muncie, 1996: 23, traducción: H/O/P).

Los problemas de fiabilidad y comparabilidad de las estadísticas criminales han motivado a los investigadores empíricos a postular que se deba recurrir preferiblemente a los registros de homicidios. Consideran que estos servirían como una especie de meta-indicador para analizar la violencia y la delincuencia en general y que permitirían hacer conclusiones válidas tanto en cuanto a países individuales como en el marco de comparaciones internacionales: "De todos los tipos de crímenes, las estadísticas de homicidios intencionales son los que menos padecen de subreportación, 
subregistro y no-uniformidad de las definiciones, $y$ la incidencia de homicidios parece ser representativa en cuanto a otros tipos de crímenes comunes" (Fajnzylber/Lederman/Loayza, 1998: 238, traducción: H/O/P). No es posible discutir aquí si un análisis serio de fenómenos sociales tan complejos como son la violencia $y$ la delincuencia realmente pueda basarse en un solo indicador estadístico, aunque sea más fiable y comparable que otros.

La socióloga Teresa Caldeira está consciente de las limitaciones de estadísticas criminales pero justifica su decisión de utilizarlas, en el caso brasileño, argumentando que las distorsiones se producen con cierta continuidad en el tiempo. Según ella, aunque no se pueda confiar en las cifras absolutas, sí servirían para identificar las dinámicas de violencia y delincuencia a grandes rasgos (Caldeira, 2000: 115). Para el caso centroamericano, en cambio, hay que cuestionar mucho más las estadísticas en comparación a las que se recopilan en los países del Cono Sur o a las referentes a las grandes metrópolis brasileñas. En Centroamérica, ciertos acontecimientos (por ejemplo, la presencia de ONUSAL en El Salvador o la introducción de políticas de "mano dura" en Honduras) y cambios importantes en la infraestructura de seguridad (por ejemplo, alteraciones en la equipamiento de la policía nicaragüense con recursos humanos y presupuestales) implican que las distorsiones estadísticas no parecen tan uniformes a lo largo del tiempo.

Aparte de las estadísticas criminales, otro indicador clave de violencia y delincuencia en muchos estudios empíricos son las encuestas. En el contexto centroamericano se recurre frecuentemente, por ejemplo, a los sondeos del Instituto CID Gallup. Dichos datos también se emplean como una de las fuentes del "Latinobarómetro" (www.latinobarometro.org). Las encuestas de CID Gallup se llevan a cabo anualmente en casi toda Latinoamérica y contienen una serie de preguntas referentes a la percepción de seguridad e inseguridad. Los resultados de las encuestas parecen indicar que la violencia constituye uno de los problemas centrales de Centroamérica (véase, por ejemplo, CID Gallup, 2004a; CID Gallup, 2004b; CID Gallup, 2005).
En la interpretación de los datos de encuesta se supone frecuentemente que estos reflejen hechos objetivos. Si esto fuese así, la violencia es uno de los problemas sociales más apremiantes de la región porque las personas encuestadas así lo afirman. Empero, se hace caso omiso a la pregunta, sin tomar en cuenta la violencia presentada en los medios de comunicación (Huhn/Oettler/Peetz, 2006) influye en las respuestas de los encuestados. Adicionalmente, en algunos casos la metodología del sondeo es cuestionable ${ }^{1}$. También es importante el considerar el uso que hacen los medios, la clase política $y$ otros actores de las (dudosas) estadísticas criminales.

Por ejemplo, con los datos de sondeos acerca de la violencia juvenil, la mayoría de los encuestados considera legítimas y apropiadas las políticas de "mano dura" contra las pandillas juveniles (véase, por ejemplo, Peetz, 2005: 355), se utilizan para documentar que existe la amenaza. Más las opiniones expresadas en las encuestas no se analizan como indicios por sí mismos de una amenaza a la democracia en Centroamérica.

En ese contexto, lo cuestionable no son tanto las encuestas como tales. El método de recolección de datos es, en la mayoría de estas encuestas, lo suficientemente transparente. Que los resultados de las encuestas se presentan como la opinión de "la población" y no como la de un sector específico de la población (personas de la clase media urbana, con teléfono, etc.) no tiene que ver con la recolección de datos sino con la interpretación de los mismos. Esas interpretaciones se efectúan muchas veces de

Por ejemplo, muchas de las encuestas se realizan por teléfono. La representatividad es cuestionable porque se excluye sistemáticamente a personas sin teléfono. Además, algunas encuestas tienden a la sobre o sub-representación de ciertos sectores de la población. Por ejemplo, Cruz (2004) recurre a datos de una encuesta realizada entre 2914 personas en El Salvador y 1200 personas en Guatemala, haciendo caso omiso de que la población total guatemalteca llega casi al doble de la de El Salvador. Además, en Guatemala se entrevistó sólo a 48 personas que se autodefinían como indígenas aunque se estima que ese sector poblacional llega a un $60 \%$ en el país. 
modo selectivo. Igual que las estadísticas criminales, las encuestas son pruebas que demuestran la severidad de la violencia y la delincuencia. Además, se evita constantemente analizar aquellas informaciones, también generadas por algunos sondeos, que ayudarían a descubrir por qué tantos de los encuestados caracterizan la violencia y la delincuencia como un problema tan grande. Una encuesta reciente en El Salvador, por ejemplo, contenía una pregunta acerca de los temas tratados en los medios de comunicación a los que los encuestados mejor se podían acordar. La mayoría recordó sobre todo noticias sobre el crimen y la violencia (CID Gallup, 2005: 7).

Caldeira (2000) recomienda no concebir a las estadísticas criminales y las encuestas como hechos objetivos sino más bien como una especie de fotografías instantáneas del discurso público:

Estadísticas criminales son construcciones que generan opiniones determinadas sobre algunos segmentos de la realidad social. Construyen imágenes de patrones del crimen y del comportamiento criminal. [...] Pero, aunque la información que las estadísticas aportan sobre el crimen es limitada, pueden sin embargo revelar otros hechos sobre la sociedad que las produce (Caldeira, 2000:106, traducción: H/O/P). [Estas reflexiones son de suma importancia para el enfoque de análisis de discurso, que ofrecería una alternativa viable para estudiar la violencia y la delincuencia en Centroamérica].

\section{LOS APORTES DEL MICROANÁLISIS}

La segunda rama de las investigaciones la constituyen micro-estudios antropológicos o sociológicos que analizan detalladamente la situación o el desarrollo de una (o varias) localidad(es) o de un grupo específico de personas $^{2}$. Sobre todo para examinar el

2 Por ejemplo en Guatemala: AVANCSO 1989 y Moser/ McIlwaine (2004); en El Salvador: Smutt/Miranda 1998, Cruz/Portillo 1998, Santacruz Giralt et ál. fenómeno de las pandillas juveniles se recurre con frecuencia a esa metodología. En estos textos se examinan las condiciones locales que influyen en las dinámicas de violencia y seguridad. Para Centroamérica es muy relevante la investigación sobre la relación entre procesos de segregación urbana, desorganización social y dinámicas de violencia (Harvey, 1988; Davis, 1990; Heitmeyer/Dollase/Backes, 1998). El punto de partida de esta corriente es en muchos casos la realidad de los barrios cerrados ("gated communities" o "ciudadelas de asentamiento"3) de los sectores acaudalados de la población. Estas residenciales protegidas $y$ vigiladas se interpretan como aceleradores de la erosión del espacio público y de la vida pública (Blakely/Zinder, 1997; Eisner, 1997). Los trabajos de Teresa Caldeira son de suma importancia en ese contexto porque relacionan analíticamente la segregación socio-espacial con el surgimiento de "discursos de miedo", con la (des)integración social y con políticas y acciones policiales violatorios a los derechos humanos. La violencia delincuencial se analiza como fenómeno ambivalente: como "experiencia desorganizativa" ("disorganizing experience") y al mismo tiempo como "símbolo organizativo" (“organizing symbol”) (Caldeira, 2000:21).

A diferencia de los estudios entre el nivel macro, los estudios sociológicos y antropológicos del nivel micro (nivel de barrio o de algún grupo específico de personas) no dependen de las estadísticas criminales ni de encuestas de opinión (supuestamente) representativas. Su base empírica son datos recolectados por los mismos investigadores; en muchos casos se trata de entrevistas cualitativas con los habitantes del respectivo barrio o de anotaciones del investigador en el marco de una observación participante.

2001, Lodewijkx/Savenije 1998, Savenije/AndradeEekhoff 2003; en Honduras: Save the Children/ACJ 2002; en Nicaragua: DIRINPRO et al. 2004 y Rodgers 2002, 2003 y 2004; en Costa Rica: Sandoval García 2006, Sanabria León 2004 y Ortiz et al. 1998 y en varios países de la región: ERIC et ál. 2001, 2004a/b y 2006 .

3 Traducción literal de la palabra alemana "Siedlungszitadellen", acuñada por Nogala (2000: 61). 
Los estudios al nivel local contribuyen con conocimientos e interpretaciones muy relevantes a la discusión sobre violencia, delincuencia y seguridad en Centroamérica. Sin embargo, su carácter local también implica ciertas limitaciones en cuanto al alcance de sus resultados. Los micro-estudios difícilmente pueden hacer afirmaciones generales acerca de toda una ciudad, un país o la región. Los datos que utilizan son demasiado limitados como para ser generalizados — salvo las excepciones que examinan una variedad más representativa de localidades, como ERIC et al. (2004) o Savenije/AndradeEekhoff (2003). Difícilmente estos estudios pueden contener afirmaciones empíricamente fundadas sobre el macro-contexto político, social o económico y su relevancia para el surgimiento de las maras- es decir, afirmaciones en cuanto a la influencia de las condiciones geográficasurbanísticas, de las consecuencias de la globalización o de las políticas de seguridad del gobierno nacional, etc.

Además, una perspectiva usualmente desatienda por los estudios locales es la dimensión discursiva del problema. Generalmente, las respuestas obtenidas en entrevistas o encuestas con la población local se presentan como manifestaciones de una realidad objetiva (una excepción es, hasta cierto grado, Moser/McIlwaine, 2004 porque la percepción constituye explícitamente el foco de la investigación). Casi nunca se analizan los orígenes ni las consecuencias del discurso que circula en las respectivas comunidades. No se examina, por ejemplo, por qué los habitantes de un barrio perciben la situación de violencia y seguridad de la manera en que la describen en sus respuestas. No se investiga cómo se construye lo que en el barrio se acepta como "la verdad" sobre esta situación. Tomando en cuenta lo que los macro y los micro-estudios contribuyen al conocimiento $y$ al debate sobre la violencia y la seguridad en Centroamérica - pero también lo que hasta el momento no han podido esclarecer- el desiderátum principal en este campo serían investigaciones que analicen el discurso tanto de los hablantes en los microcosmos locales como de los hablantes (relativamente poderosos) en el macrocosmos de la política, de los medios de comunicación, etc. Este análisis tendría que contextualizar el respectivo discurso con las condiciones y los procesos relevantes de los niveles local, nacional, regional y global.

\section{NEGOCIANDO INSEGURIDADES: LA CONSTRUCCIÓN DE LA REALIDAD VIOLENTA}

Aparte de los problemas metodológicos inherentes al uso de estadísticas criminales y encuestas, parece aún más importante señalar otro déficit de los estudios empíricos y sobre todo de su papel dominante en el debate sobre los procesos sociales en Centroamérica. Se tiende a desatender el hecho de que las interpretaciones y clasificaciones sociales son construcciones sociales, $y$, por ende, productos inestables de luchas discursivas. Así, muchos estudios empíricos reflejan un concepto estático del mundo social sin tomar en cuenta la acción discursiva y el dinamismo colectivo. Aunque puedan hasta cierto grado medir y cuantificar la violencia $y$ la delincuencia, contribuyen muy poco al análisis de lo que estos fenómenos significan socialmente o al análisis de los procesos sociales que supuestamente son reacciones a estos fenómenos.

Partiendo de la noción de que la realidad se construye socialmente (Berger/Luckmann, 1969) hay que interpretar a la violencia y delincuencia como construcciones sociales y no como fenómenos objetivamente existentes. En la mayoría de los estudios empíricos se presupone un consenso tanto en cuanto a la existencia objetiva y a la definición de la violencia $y$ delincuencia como en cuanto a su valoración (negativa) por la sociedad. De ese modo se eluden preguntas difíciles pero claves: ¿Qué es exactamente lo que se percibe como amenaza? ¿de qué manera y por qué se perciben esas amenazas? "La categorización de una acción violenta es sujeto al cambio histórico y cultural $y$, sobre todo, es objeto de conflictos sociales $y$ culturales" (Liell, 2002: 6, traducción: H/O/P). La violencia física se puede evaluar de maneras muy diferentes por una sociedad y correspondiendo a eso se define como problema más o menos grave. La violencia, en perspectiva de sentido social, no simplemente existe como hecho objetivo. Se percibe y se evalúa de forma 
diferente según su contexto histórico, social y cultural. Por ejemplo, un puñetazo se puede interpretar socialmente como acto de violencia ilegítima. En otro contexto la misma acción puede categorizarse como hazaña en una competición deportiva, como acto tolerable en la adolescencia de un hombre o como acción legítima en la lucha por un objetivo mayor o una utopía. Matar a una persona se puede considerar simplemente un crimen o, en otro contexto histórico, un acto imprescindible $-y$ por lo tanto aceptable - en la consecución de un fin social (por ejemplo, una guerra).

Recientemente Wieviorka (2006: 210) recordó que, sobre todo en la Europa de los 70's y 80's y en el marco de algunas utopías, a la violencia se le concedía cierta legitimidad y muchos intelectuales la defendían o la justificaban. Hoy en día, en cambio, la violencia se ha convertido en símbolo indiscutible y ubicuo del mal. Para Centroamérica se puede constatar una reevaluación parecida desde el fin de la época de las revoluciones y utopías sociales de los años 50 hasta los 80 . No existía un consenso tan amplio de rechazo rotundo a la violencia como recurso político. Tampoco había tal consenso en cuanto a (ciertas formas de) la delincuencia.

De estos procesos de definición y construcción social depende qué es lo que se considera como delincuencia en una sociedad, sea esta, delincuencia violenta o no violenta. En perspectiva sociológica, más importante que el acto de violencia en sí es la asignación social de sentido. Igual que todos los fenómenos sociales, la violencia y la delincuencia sólo se vuelven reales cuando la sociedad las percibe, las denomina, las clasifica y las reconoce (como reales). Sirva como otro ejemplo la violencia domestica ${ }^{4}$. En el marco de un proceso social el fenómeno se convirtió en un tema

Los autores utilizan el término "violencia doméstica" conscientes de que es impreciso porque se refiere a un tipo de violencia relacionado con un lugar - la casa (Lang 2004 llama la atención a ese problema terminológico). El término "violencia intrafamiliar" tampoco es preciso ya que excluye los actos de violencia en parejas que no se consideran familia. público, después se generó un amplio consenso de juzgarlo como negativo y de proscribirlo. Finalmente se penalizó convirtiéndose así en delito. Este proceso no se puede examinar cabalmente con una metodología empírica, ni se puede investigar con ella satisfactoriamente la argumentación hegemónica en que se basó este proceso. Es decir, con un enfoque exclusivamente empírico no es posible descifrar el discurso en el cual el descrito proceso social se fundamentó. Pero desde el punto de vista de las ciencias sociales es justamente el análisis de este discurso, el que permitiría una aproximación a lo esencial del fenómeno.

La forma en que una sociedad percibe $y$ reacciona ante la violencia y la delincuencia depende más de los procesos dentro de esta sociedad para "negociar" la definición y el sentido de la violencia y la delincuencia — es decir, depende más del discurso sobre estos fenómenos-que de los actos de violencia o delincuencia como tales. La percepción de (in)seguridad y las reacciones colectivas frente a la violencia y la criminalidad (por ejemplo el aumento de penas, la intensificación de la prevención o la privatización de la seguridad, pero también prácticas solidarias e integrativas que contribuyen a la seguridad) son, en primer lugar, consecuencias y simultáneamente partes de o contribuciones a un discurso. Sólo en un segundo plano son resultados de la violencia y delincuencia en sí. Por ejemplo, después de la introducción de las políticas de "mano dura" contra las maras en el año 2003 en El Salvador, el 52,3 \% de los entrevistados por IUDOP (2004: 43) en el año siguiente respondió tener la impresión de que la delincuencia había disminuido. Sin embargo, tanto el gobierno como los medios de comunicación seguían refiriéndose a las pandillas juveniles como amenaza creciente $y$ legitimaban con eso la continuación de las políticas represivas de seguridad. El miedo a la violencia y delincuencia no necesariamente corresponde con las estadísticas. Garland demuestra para los EEUU y Gran Bretaña que no hay una correlación directa entre el miedo y las cifras de criminalidad:

Las encuestas de opinión pública desde los años 1970 muestran que la mayoría de la gente cree que el problema de la 
delincuencia es grave y empeora y que la criminalidad irá en aumento en el futuro: esa opinión persiste incluso en épocas cuando las tasas de delitos, tanto registrados como cometidos, están estables o bajando (Garland, 2003: 107, traducción: $\mathrm{H} / \mathrm{O} / \mathrm{P})$.

El ejemplo dado indica que no son los hechos los que más influyen en las opiniones sino más bien los discursos y las prácticas sociales.

Está comprobado que sentirse seguro depende fuertemente de medidas públicas (como la intensificación de controles o el aumento del presupuesto de seguridad) y de creer o no que las agencias encargadas de la seguridad tienen la capacidad de mejorar la situación (véase, por ejemplo, Garland, 2003: 122). Además, el miedo ante algún tipo de delito puede ser muy grande en la población aunque este crimen se cometa estadísticamente pocas veces. "El tratamiento sensacionalista de la violencia $y$ de hechos delictivos puede generar un clima de miedo y una sensación fuerte de vulnerabilidad entre la población, que no siempre es real o correspondiente al nivel de violencia observado" (Arriagada/Godoy, 1999: 10, traducción: H/O/P). Por ejemplo, dadas las cifras relativamente bajas de los homicidios en Costa Rica, el miedo en la población ante este delito parece desproporcionado (Córdoba, 2006: 12-13). En este caso el miedo no se basa en estadísticas de homicidios sino en un discurso público que se materializa sobre todo en los periódicos y la televisión donde los reportajes sobre asesinatos tienen una cobertura muy amplia (Córdoba, 2006: 13). La criminología crítica, la cuál se enfoca en las instituciones con capacidad de criminalización, ha subrayado el papel de los medios de comunicación que desvían la atención pública en tiempos de crisis social (Baratta, 1986; Scheerer, 1978). En este contexto también se podrían discutir las motivaciones de grupos de interés que se benefician con la sensación de inseguridad de la población. Estos grupos pueden ser, entre otros, líderes políticos que esperan obtener ganancias electorales por pronunciarse a favor de la "mano dura" o empresarios de seguridad privada quienes — como constata Nils Christie
(2000: 138iss) - tienen que procurar que el mercado no parezca saturado para sus productos y servicios.

Pero el actuar del estado y la percepción colectiva de crisis tampoco están relacionados de forma directamente causal. Más bien, parece haber una relación de interdependencia y de intensificación mutua y hasta podría ser que se originan paralelamente. Para analizar la significación y las consecuencias de la violencia y de la delincuencia hay que investigar los discursos $y$ sus contextos sociales - en vez de postular la existencia (creciente) de la violencia valiéndose, como es común, del meta-indicador de los homicidios y definiendo la violencia como algo objetiva y eternamente mala.

La pregunta clave, por lo tanto, no concierne al grado de violencia "medido" en un país o en una región. Más bien, la interrogante central es: ¿cuáles son los discursos de violencia que circulan, en cuáles espacios públicos, y cuáles son las prácticas sociales y políticas relacionadas con estos discursos? Como recomienda Caldeira (2000), los datos estadísticos tienen que interpretarse como reflejos de discursos de violencia y como reflejos de los procesos sociales originados en el marco de estos discursos, pero no como una realidad objetiva. Partiendo del postulado del "doble vínculo" (Verón, 1996: 126) hay que constatar que las estadísticas criminales centroamericanas - como elementos del discurso de violencia- reflejan, pero, al mismo tiempo, crean los fenómenos que se intentan medir con ellas.

\section{LA IMPORTANCIA ANALÍTICA DEL DISCURSO COMO PRÁCTICA SOCIAL}

El discurso, o mejor dicho, los discursos de violencia y delincuencia constituyen el trasfondo de la violencia percibida en la población. La existencia y el aumento de esta violencia se postulan públicamente, los medios de comunicación la dramatizan y los órganos del estado la combaten. Con esta afirmación no se pretende negar que halla violencia $y$ delincuencia en los países centroamericanos. Sin embargo, la hipótesis es que el potencial amenazante que se adjudica a la violencia y delincuencia es 
una construcción social. Las reacciones de las sociedades a la violencia y delincuencia - sea el endurecimiento de las penas y de la persecución del delito, sea la desintegración social en las ciudades provocada por un urbanismo segregativo y por el uso extensivo de tecnologías de protección- son consecuencia de esta construcción social y no de la violencia que se mide estadísticamente. Hay que diferenciar en este contexto entre el individuo y la sociedad. La víctima de un acto de violencia, de hecho, reacciona a este acto ( $y$ no a un discurso o a una construcción social). Pero como procesos sociales las reacciones son parte y consecuencia de la organización social y de interpretaciones colectivas. La relación entre el discurso de violencia y la violencia es dialéctica, igual que la relación entre el discurso de violencia y el consenso o el conflicto (en una sociedad, en un momento histórico determinado) acerca de cómo reaccionar frente a la violencia. También existe una relación dialéctica entre el discurso de violencia y el statu quo de la autodefinición de una sociedad. "El discurso contribuye a la constitución de la sociedad y la sociedad genera $y$ determina el discurso" (Fairclough/Wodak, 1997: 258, traducción: H/O/P).

Un discurso se genera en numerosos espacios discursivos en los que diferentes actores compiten por la definición e interpretación de varios fenómenos particulares. Aunque sea común creer que actores individuales, como los políticos o los medios masivos de comunicación, "crean" opiniones (aceptadas tal cual por la sociedad), parece más razonable el postulado de Jäger de que un discurso es poco controlable:

El discurso es creado por el conjunto de todos los individuos. Pero los individuos participan de forma desigual en las diferentes vías discursivas. Los individuos tienen diferentes márgenes de maniobra asignados a ellos por los discursos sociohistóricos preexistentes. Sin embargo, ninguno de los individuos determina el discurso. El discurso es, por así decirlo, el resultado del total del sinnúmero de esfuerzos de los seres humanos para actuar dentro de una sociedad. Este resultado es algo que nadie ha querido así, pero que todos han contribuido de diferentes maneras $y$ en diversos ámbitos de sus vidas ( $y$ con diferente peso). (Jäger, 2004: 148, traducción H/O/P).

[Pero esta auto-dinámica que desarrollan los discursos no significa que se generen de manera caótica].

Los autores de la corriente del Análisis crítico del discurso tienen toda la razón en destacar que la producción y las condiciones de producción de un texto - es decir, de una contribución a un discurso- se deben tener tanto en cuenta como el texto en sí. Los discursos no se pueden analizar de manera sensata sin considerar su contexto de poder, historia e ideología. Estos factores determinan qué tan "natural" parece una construcción social, como en este caso la violencia y la delincuencia, en una sociedad $y$ definen cómo $y$ hasta qué grado es posible romper las convenciones (Wodak, 2001: 3; véase también van Dijk, 1999).

Para examinar el discurso sobre violencia, criminalidad, inseguridad, vulnerabilidad etc. hay que desmembrarlo; Foucault (1997) utilizaba el término "arqueología" para describir sus exploraciones. La primera pregunta es dónde buscar los actos de habla o los fragmentos del discurso y quiénes son los hablantes y los actores. En el caso del discurso de violencia en Centroamérica, los espacios más importantes donde se desarrollan los diferentes ramos del discurso son: la política, la justicia, los medios masivos de comunicación, las ciencias sociales y "la vida cotidiana". En los siguientes párrafos se explicará por qué parece fructuoso investigar y "hacer arqueología" — como diría Foucault - en estos cinco espacios sociales. La política en general es un escenario importante. En ese ámbito se negocia el reconocimiento de prácticas sociales y la evaluación y legitimidad de estas prácticas. El principio del monopolio de violencia del estado hace que sea este el ámbito político donde las prácticas se transforman en prácticas reconocidas y legítimas. Aparte de políticos (profesionales) como tales los actores $y$ hablantes en ese campo también son personas y grupos de la sociedad civil. Un área estrechamente vinculada a la de la política es la de la justicia. Para el discurso de violencia 
son muy relevantes tanto la definición de lo legal y lo ilegal (leyes) como las prácticas jurídicas y la aplicación de las leyes.

Otro ámbito importante para la construcción social de la realidad (violenta) son los medios masivos de comunicación. Ellos no sólo representan y multiplican opiniones; también las producen y las transforman (Bourdieu, 1998: 28). Los medios son una plataforma para la presentación de las opiniones de hablantes (periodistas, pero también políticos, actores de la sociedad civil, intelectuales, etc.). Pero como instituciones con fines de lucro $y$ con su propia agenda política los medios son al mismo tiempo actores (en muchos casos: actores poderosos). Cocco (2003: 57) se refiere a esa doble función de los medios con la observación de

... que más allá de ser una reproducción de la realidad, la noticia es una creación. Los noticieros imitan, pero también crean - crean nueva realidad, reorganizan el mundo y la cotidianidad, dándole sentido. Informar no es solo transmitir, sino dar forma e infundir significación.

También hay que considerar el campo de las ciencias porque ahí es donde se originan las bases argumentativas para diferentes fragmentos del discurso. Nuestra crítica de la precaria fundamentación empírica de muchos estudios sobre violencia en Centroamérica es parte de esa consideración.

Un discurso sólo puede desarrollar sus poderosos efectos si alcanza reconocimiento en la sociedad. Por eso es preciso investigar cuáles de los elementos del discurso público sobre violencia e inseguridad se absorben y se reproducen entre las personas que no ocupan posiciones de poder en la sociedad porque los elementos discursivos, de esa manera reconocidos, constituyen el "conocimiento válido" (Jäger, 2004: 149). Sin embargo, personas y grupos no poderosos no sólo deben considerarse como reproductores de los actos de habla de hablantes poderosos. Más bien, hay que tomar en serio las experiencias y opiniones de los "grupos dominados" (van Dijk, 2001: 96). Ahora bien, en estos diferentes ámbitos (la política, la justicia, los medios, la ciencia y la "vida cotidiana") no se producen discursos separados unos de otros. Más bien, los hablantes de todos estos ámbitos contribuyen a un solo discurso: el discurso social sobre violencia, delincuencia e (in)seguridad.

Para indagar en los discursos que circulan en estos espacios sociales (espacio político, jurídico, mediático, científico y "cotidiano") es necesario recurrir a fuentes que reflejen los respectivos discursos. Por ejemplo, en el marco del proyecto "Espacios públicos y violencia en Centroamérica" del GIGA Institute of Latin American Studies, Hamburgo/Alemania, los autores de este artículo han recolectado programas y manifiestos de partidos políticos, declaraciones de líderes políticos, leyes $y$ otros textos legales, artículos de prensa, textos científicos, unas 90 entrevistas cualitativas con una amplia gama de personas (de diferentes clases sociales, profesiones, sexos y edades). Además, para captar el discurso cotidiano, se ha pedido a unos 230 estudiantes de secundaria redactar breves textos acerca de la temática.

La segunda pregunta importante para desmembrar ese discurso es sumamente difícil: ¿Cuáles contenidos, temas y categorías se pueden diferenciar dentro del discurso y cuáles son los motivos de los hablantes y cuáles los contextos en los que se enmarcan sus actos de habla? (Meyer, 2001: 15). Con "motivos" y "contextos" se refiere, una vez más, a cuestiones de poder, historia e ideología y no a la argumentación de los hablantes. El discurso de violencia se refiere muy pocas veces a la violencia como categoría general $y$ abstracta. Más bien, trata generalmente de fenómenos y formas específicos de la violencia, como son la violencia familiar o la violencia juvenil.

En este contexto, otra pregunta es si hay un consenso sobre los contenidos del discurso. Un contenido destacado del discurso de violencia en Centroamérica en general es, por ejemplo, la violencia juvenil. Sobre este fenómeno se discute en todo el istmo aunque el debate tenga distintas características y enfoques en los diferentes países. Se señala que la violencia juvenil está incrementando. "Esto es cierto tanto para referirse a las maras callejeras, extremadamente violentas, que operan en los países de Guatemala, El Salvador y Honduras, como para referirse a las pandillas de barrio que caracteriza a la violencia juvenil en Nicaragua" (Cruz, 2006: 406). 
CONCLUSIÓN: LA NECESIDAD DE ANALIZAR EL TALK OF CRIME

En las páginas anteriores se ha expuesto un panorama teórico muy distinto $y$ discrepante de los policy papers de los mayores think tanks dedicados al estudio de la gobernabilidad y gobernanza. El análisis aquí presentado, que enfatiza la creación, divulgación, consolidación e institucionalización de los discursos sobre violencia en Centroamérica, tiene la intención de poner en debate las concepciones vigentes en torno a la inseguridad ciudadana. Centroamérica, una región sumamente heterogénea, se ha caracterizado en su historia reciente por marcadas transformaciones en cuanto a la percepción de la (in)seguridad. Como se pudo ver en las páginas anteriores la relevancia de cada uno de los diferentes fenómenos de violencia varía considerablemente entre los cinco países ${ }^{5}$.

En El Salvador y Honduras los problemas de las maras y de la proliferación de armas son considerados en la prensa escrita, dos temas tan destacados que otros temas de violencia casi parecen no tener importancia. En Guatemala junto con el pandillismo los temas centrales son los feminicidios, los linchamientos y la violencia estatal y para-estatal en el marco del "Estado de Mafia Corporativa" ("corporate mafia state", expresión acuñada por Amnistía Internacional). En Nicaragua la atención pública se centra, aparte de la corrupción, en la doméstica, en el narcotráfico, en la violencia en el contexto de la migración (especialmente violencia en contra de emigrantes nicaragüenses en Costa Rica), y en algo que se podría resumir como violencia y brutalidad cotidiana. En Costa Rica, lo que más se discute es la violencia y brutalidad cotidiana, la narco-delincuencia, la violencia doméstica y la delincuencia menor. En ese país frecuentemente se intenta buscar o construir una relación entre la delincuencia y la violencia con la inmigración masiva de nicaragüenses (véase, Sandoval, 2006b).

5 Para El Salvador, Nicaragua y Costa Rica véase el análisis de la prensa escrita en Huhn/Oettler/Peetz 2006.
A pesar de esa heterogeneidad hay dos aspectos importantes compartidos por los cinco países:

La violencia y la delincuencia juegan un papel clave en las percepciones cotidianas $y$ en los discursos sociales. En ese contexto se producen cambios en cuanto a los sistemas de valores de las sociedades, en cuanto a la aceptación de la democracia $y$ del principio del estado de derecho, en cuanto a la convivencia social y también en cuanto a la percepción de los países vecinos y de la región.

Los esfuerzos por solucionar otros problemas centrales de las sociedades - como la pobreza, la corrupción o el acceso inequitativo a los recursos económicos, culturales etc.- pasan cada vez más a un segundo plano, o bien se dejan de hacer por completo, en beneficio de la concentración de recursos humanos y financieros en el área de seguridad. Esto no sólo respecta al contexto nacional. La cantidad de recursos que se están invirtiendo en el combate conjunto de las maras (sobre todo de parte de El Salvador, Guatemala y Honduras), en el control de las fronteras y de la migración $y$ en la lucha contra el narcotráfico indican que se trata de procesos $y$ discursos hasta cierto grado regionales. En los cinco países los diferentes fenómenos de la violencia también son vistos como una consecuencia natural de los altos grados de pobreza y desigualdad social. En Nicaragua y en menor medida en Costa Rica prevalecen esfuerzos preventivos para solucionar el problema de la violencia. En Guatemala, El Salvador y Honduras predominan esfuerzos represivos.

El talk of crime está muy presente en todos los países del istmo (véase también Huhn/ Oettler/Peetz 2006). En espacios discursivos políticos, mediáticos, jurídicos, científicos y cotidianos la violencia y la delincuencia se convierten en temas cada vez más centrales. La tendencia general parece ser que la inseguridad se va agravando.

No se pretende decir que la violencia en Centroamérica solamente sea ficción. Tampoco hay que dudar tan fundamentalmente de 
las estadísticas como para cuestionar que la violencia es un problema "real" y de mucha relevancia. Ni mucho menos se tiene la intención de desvalorar o menospreciar el miedo, la preocupación o la rabia que la violencia y la delincuencia producen en muchos centroamericanos. Pero en todos los países de la región se está llevando a cabo una lucha de definición, interpretación y clasificación entorno al campo temático "violencia/delincuencia/(in)seguridad". Y aunque esta lucha tenga consecuencias políticas y sociales sumamente relevantes para las sociedades centroamericanas, no ha sido objeto de un análisis sistemático (sólo se han examinado unos aspectos muy específicos del tema; véase, por ejemplo, Fonseca Vindas/ Sandoval García, 2006). Muchos estudios sobre Latinoamérica y Centroamérica destacan la importancia y el poder de los discursos sociales (por ejemplo Berardi, 2005; Caldeira, 2000; Cocco, 2003; Fonseca Vindas/Sandoval García, 2006; Sandoval García, 2002; para sólo mencionar algunos). Mas un análisis comprensivo sobre un problema clave de la realidad social en Centroamérica —el talk of crime centroamericano- aún no se ha elaborado.

La "mano dura" en El Salvador, Honduras y Guatemala, la privatización de la seguridad y la segregación espacial en todos los países de la región, igual que muchos intentos comunitarios $y$ a veces muy solidarios de parte de un creciente número de ongs que trabajan este tema son ejemplos para los cuantiosos procesos sociales que se legitiman como medidas para contrarrestar la violencia y la delincuencia. Lo que hace falta es examinar dentro de sus respectivos contextos los discursos que están interrelacionados dialécticamente con estos procesos. Desde esta perspectiva parece poco prometedor recurrir a las estadísticas criminales; $y$ en cuanto a la cobertura mediática Fonseca Vindas/Sandoval García (2006: 33) constatan que

[la] sensación de inseguridad no solo es consecuencia del aumento de hechos delictivos, sino también del incremento de la oferta de los medios en material de sucesos y del surgimiento de programas, especialmente televisivos, cuyo tema preferido son los sucesos.
¿Cuáles son las causas de la violencia y de la inseguridad desde el punto de vista de los diferentes hablantes y actores? ¿Cuáles son las estrategias de solución del problema que proponen o que implementan los hablantes y actores y cuáles son sus argumentos? ¿Cuáles de las reacciones de las sociedades a la violencia e inseguridad aceptan y cuáles critican? ¿Y cuáles son los efectos de estas reacciones en cuanto a los sistemas de valores de cada sociedad? Investigaciones, que tratasen de los discursos sobre la violencia, revelarían en qué espacios públicos se sitúan los discursos sobre diferentes fenómenos de violencia y sobre las opciones de reducir o contenerlos. De tal modo, se podría detectar una visión supranacional que contempla la divulgación de discursos represivos, los cuales -en el marco de transformaciones socioeconómicas fundamentales- aceleran procesos de segregación social $y$ contribuyen a socavar los fundamentos del Estado de derecho y de la democracia.

\section{BIBLIOGRAFÍA}

Arriagada, Irma; Godoy, Lorena. "Seguridad ciudadana y Violencia en América Latina: diagnóstico y políticas en los años noventa". Social Policy Series 32. Santiago de Chile: CEPAL, 1999. En: <www.eclac.org/ publicaciones/xml/7/4657/lcl1179e.pdf>. [Consultado el 23/10/2006].

\section{Baratta, Alessandro. Criminología crítica y crítica del derecho penal. Ciudad de México: Siglo XXI, 1986.}

Berardi, Leda (ed.). "Análisis crítico de discurso". Perspectivas Latinoamericanas. Santiago de Chile: Frasis, 2005.

Berger, Peter; Luckmann, Thomas. Die gesellschaftliche Konstruktion der Wirklichkeit. Eine Theorie der Wissenssoziologie. Frankfurt/M.: Fischer Verlag, 1969.

Blakely, Edward J.; Snyder, Mary G. Fortress America. Gated Communities in the 
United States. Washington D.C.: Brookings Institution Press, 1997.

Bourdieu, Pierre. Über das Fernsehen. Frankfurt/M.: Suhrkamp, 1998.

Caldeira, Teresa P.R. City of Walls. Crime, Segregation, and Citizenship in São Paulo. Berkeley/Los Angeles/London: University of California Press, 2000.

Christie, Nils. Crime Control as Industry: Towards Gulags Western Style. London: Routledge, 2000.

CID Gallup. Public Opinion Poll 39. Guatemala, San José: CID Gallup. 2004a. En: <http:// www.cidgallup.com/docs/opiniones/ Gua39ebrief.pdf $>$, [Consultado el 8/8/2007].

. Public Opinion Survey 57. El Salvador, San José: CID Gallup. 2004b. En: <www. cidgallup.com/archivos/BOLES57e.pdf $>$. [Consultado el 23/10/2006].

. Public Opinion Survey 58 El Salvador, San José: CID Gallup. 2005. En: <http:// www.cidgallup.com/docs/opiniones/ OP58SALe.pdf>. [Consultado el 8/8/2007].

Cocco, Madeline. "La identidad en tiempos de globalización. Comunidades imaginadas, representaciones colectivas y comunicación”. Cuadernos de Ciencias Sociales. San José: FLACSO, 2003.

Córdoba, Javier. "Medios de comunicación fomentan la violencia y el miedo". Inforpress Centroamericana. 30/6/2006.

Cruz, José Miguel. "Violencia y democratización en Centroamérica: El impacto del crimen en los regímenes de posguerra, San Salvador". "América latina hoy". Revista de Ciencias Sociales 35. 2003: 19-59. En : $<w w w . v i o l e n c i a e l s a l v a d o r . o r g . s v / i m a g e s /$ stories/documentos/aportes_para_la_ convivencia_y_la_seguridad_ciudadana/15. pdf $>$, continúa en $<w w w . v i o l e n c i a e l s a l v a d o r$. org.sv/images/stories/documentos/ aportes_para_la_convivencia_y_la_ seguridad_ciudadana/16.pdf $>y<w w w$. violenciaelsalvador.org.sv/images/ stories/documentos/aportes_para_la_ convivencia_y_la_seguridad_ciudadana/17. pdf $>$. [Consultado el 25/10/2006].

Cruz, José Miguel (ed.). "Maras y pandillas en Centroamérica". Las respuestas de la sociedad civil organizada IV. San Salvador: UCA Editores, 2006

Cruz, José Miguel / Portillo, Peña. Solidaridad y violencia en las pandillas del gran San Salvador, más allá de la vida loca. San Salvador: UCA Editores,1998.

Davis, Mike City of Quartz: Excavating the Future in Los Angeles. London: Verso, 1990.

DIRINPRO, NITLAPAN, IDESO. Muerte arriba. Las pandillas en Nicaragua 1999-2004. Managua: UCA Publicaciones, 2004.

Eisner, Manuel. Das Ende der zivilisierten Stadt? Die Auswirkungen von Modernisierung und urbaner Krise auf Gewaltdelinquenz. Frankfurt/M.: Campus, 1997.

ERIC/IDESO/IDIES/IUDOP (eds.). Maras $y$ pandillas en Centroamérica I. Managua: UCA Publicaciones, 2001.

(eds.). "Pandillas y capital social". Maras y pandillas en Centroamérica II. San Salvador: UCA Editores, 2004a.

ERIC/IDIES/IUDOP/NITLAPAN/DIRINPRO (eds.). "Políticas juveniles y rehabilitación". Maras y pandillas en Centroamérica III. Managua: UCA Publicaciones, 2004b.

Fairclough, Norman; Wodak, Ruth. "Critical Discourse Analysis". van Dijk, Teun A. (ed.) Discourse as Social Interaction: Discourse 
Studies: a Multidisciplinary Introduction II. London. Sage, 1997: 258-284.

Fonseca Vindas, Karina; Sandoval García, Carlos. "Medios de communicación e (in)seguridad ciudadana en Costa Rica". Cuadernos de Desarrollo Humano del PNUD. San José, Costa Rica: Programa de las Naciones Unidas para el Desarrollo, 2006.

Foucault, Michel. Archäologie des Wissens. Frankfurt/M.: Suhrkamp, 1997.

Garland, David. The Culture of Control. Crime and Social Order in Contemporary Society. Oxford: Oxford University Press, 2003.

Harvey, David. Social Justice and the City. Oxford: Basil, 1988.

Heitmeyer, Wilhelm; Dollase, Rainer; Backes, Otto (eds.). Die Krise der Städte. Frankfurt/M.: Suhrkamp, 1998.

Huhn, Sebastián; Oettler, Anika; Peetz, Peter. "La telaraña de los discursos sobre violencia en Centroamérica". Iberoamericana 19. 2005: 188-193. En: <www.iberoamericana. de/articulos-pdf/19-huhn_et_all.pdf $>$. [Consultado en 23/10/2006].

. "Exploding Crime? Topic Management in Central American Newspapers". GIGA Working Paper 33. Hamburg. 2006. En: $<w w w$.giga-hamburg.de/workingpapers: http://www.giga-hamburg.de/dlcounter/ download.php?d=/content/publikationen/ pdf/wp33_huhn-oettler-peetz.pdf>.

Huhn, Sebastian; Oettler, Anika. "Jugendbanden in Zentralamerika: Zur Konstruktion einer nicht-traditionellen Bedrohung". Gabbert, Karin et ál. (eds.): Jahrbuch Lateinamerika 30. Mit Sicherheit in Gefahr, Münster. Westfälisches Dampfboot, 2006: 31-48.

IUDOP. "Encuesta de evaluación del año 2004. Consulta de opinión pública de noviembre de 2004". Serie de Informes 107. San Salvador: UCA Publicaciones, 2004. En: $<$ http://www.uca.edu.sv/publica/iudop/ Web/2004/informe107.pdf> [Consultado el 8/8/2007].

Jäger, Siegfried. Kritische Diskursanalyse. Eine Einführung. Münster: Unrast-Verlag, 2004

Lang, Miriam. "Privatsache oder gesellschaftliches Problem? Gewalt gegen Frauen und staatssozialistische Frauenpolitik in Kuba". Lateinamerika Analysen 8. 2004: 3-38.

Liell, Christoph. "Gewalt in modernen Gesellschaften-zwischen Ausblendung und Dramatisierung". Aus Politik und Zeitgeschichte 44. 2002: 6-13.

Lodewijkx, Hein; Savenije, Wim. "Aspectos expresivos e instrumentales de la violencia entre las pandillas juveniles salvadoreñas: una investigación de campo". Ramos, Carlos Guillermo (ed.): America Central en los Noventa: Problemas de juventud. San Salvador. FLACSO, 1998: 115-150.

MacDonald, Ziggy. "Official Crime Statistics: Their use and interpretation". The Economic Journal 112. 2002: 85-106.

Maguire, Mike. Crime statistics. The "data explosion" and it's implications. Maguire, Mike; Morgan, Rod; Reiner, Robert (eds.). The Oxford Handbook of Criminology, Oxford. Oxford University Press, 2002: 322-375.

Meyer, Michael. "Between theory, method, and politics: positioning of the approaches to CDA". Wodak, Ruth; Meyer, Michael (eds.). Methods of Critical Discourse Analysis. London. Sage Publications, Thousand Oaks, New Delhi, 2001: 14-31.

Moser, Caroline; McIlwaine, Cathy. Encounters with Violence in Latin America. Urban poor perceptions from Columbia and Guatemala. New York/London: Routledge, 2004. 
Muncie, John. "The Construction and Deconstruction of Crime". Muncie, John; McLaughlin, Eugene (eds.). The Problem of Crime. London. Sage Publications/ Thousand Oaks/New Delhi, 1996: 5-63.

Nogala, Detlef. "Gating the Rich-Barcoding the Poor: Konturen einer neoliberales Sicherheitskonfiguration". LudwigMayerhofer, Wolfgang (ed.). Soziale Ungleichheit, Kriminalität und Kriminalisierung. Opladen: Leske + Budrich, 2000.

Ortiz C., Maritza; Zamora, Alicia; Rodríguez, Ana; Chacón, Laura; Gutiérrez, Ana Lucía. "Soy una mujer de ambiente..." Las mujeres en prostitución y la prevención del VIH/SIDA. San José: EUCR, 1998.

Peetz, Peter. "Las "maras": el pandillismo juvenil en Honduras, El Salvador y Guatemala". Potthast, Barbrara; Carreras, Sandra (eds.). Entre familia, sociedad y Estado: niños y jóvenes en América Latina. Madrid. Iberoamericana/Franfurt/M. Vervuert, 2005: 333-372.

Rodgers, Dennis. "We live in a State of Siege': Violence, Crime, and Gangs in Post-Conflict Urban Nicaragua". LSE/DSI Working Paper 02-36. London: LSE, 2002. En: <www.lse. ac.uk/collections/DESTIN/pdf/WP36.pdf $>$. [Consultado el 19/9/2006].

"Dying for It: Gangs, Violence, and Social Change in Contemporary Urban Nicaragua”. Crisis States Programme Working Paper 35. London: LSE, 2003. En:

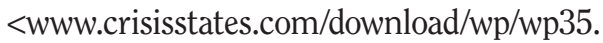
pdf $>$, [Consultado el 23/10/2006].

Disembedding the city: Crime, Insecurity, and Spatial Organisation in Managua, Nicaragua. LSE/DSI Working Paper 04-50. London: LSE, 2003. En: $<w w w . l s e . a c . u k / c o l l e c t i o n s / D E S T I N / p d f /$ WP50.pdf>. [Consultado e 19/9/2006].
Sanabria León, Jorge R. "Autonomía y prospección en adolescentes víctimas de explotación sexual". Cuadernos de Ciencias Sociales 133. San José: FLACSO, 2004.

Sandoval García, Carlos. Otros amenazantes. Los nicaragüenses y la formación de identidades en Costa Rica. San José: EUCR, 2002.

Sandoval García, Carlos (coord.). "Un mundo de colores: niños y niñas dibujan La Carpio". 2006. En: <http://iis.ucr. ac.cr/pagWeb/jornadas/Ponencias/ Ponencia\%20_de_Carlos_Sandoval. pdf\#search $=\% 22 \% 22$ un $\% 20$ mundo $\% 20$ de $\% 20$ colores $\% 22 \% 20$ sandoval $\% 22>$. [Consultado el 19/9/2006].

Santacruz Giralt, María; Alberto ConchaEastman; Homies Unidos. Barrio adentro. La solidaridad violenta de las pandillas juveniles. San Salvador: IUDOP, 2001.

Save the Children; ACJ (eds.). Las maras en Honduras: investigacion sobre pandillas y violencia juvenil, consulta nacional, propuesta de programa nacional de atencion, ley especial. Tegucigalpa: ACJ, 2002.

Savenije, Wim; Andrade-Eekhoff, Katharine. Conviviendo en la orilla. Violencia y exclución social en el Area Metropolitana de San Salvador. San Salvador: FLACSO, 2003.

Scheerer, Sebastian. "Der politischpublizistische Verstärkerkreislauf". Kriminologisches Journal 10. 1978: 223 227.

Schmidt, Daniel. Statistik und Staatlichkeit. Wiesbaden: VS, Verlag für Sozialwissenschaften, 2005.

Smutt, Marcela; Miranda, Jenny. El fenómeno de las pandillas en El Salvador. San Salvador: FLACSO, 1998. 
van Dijk, Teun A. "El análisis crítico del discurso". Anthropos 186. 2001: 23-36. En: <www. discursos.org/Art/El\%20an\%E1lisis\%20 cr\%EDtico\%20del\%20discurso.pdf >. [Consultado el 27/10/2006].

"Multidisciplinary CDA: a plea for diversity". Wodak, Ruth; Meyer, Michael (eds.). Methods of Critical Discourse Analysis. London. Sage/Thousand Oaks/ New Delhi, 2001: 95-120.

von Hofer, Hanns. "Crime Statistics as constructs: the case of Swedish rape statistics". European Journal on Criminal Policy and Research 8. 2000: 77-89.

Wieviorka, Michel. Die Gewalt. Hamburg: Hamburger Edition, 2006.
Wodak, Ruth. "What is CDA about-a summary of its history, important concepts and its developments". Wodak, Ruth; Meyer, Michael (eds.). Methods of Critical Discourse Analysis. London. Sage/Thousand Oaks/ New Delhi, 2001: 1-13.

WHO. World Report on violence and health. Geneva: WHO, 2002.

Zaffaroni, Eugenio Raúl. "Política criminal y derechos humanos en América Latina: de la 'Seguridad Nacional' a la 'Seguridad Ciudadana"'. ILANUD (ed.). Consideraciones en torno a una nueva política criminal en Centroamérica y Panamá. San José: ILANUD, 1992. 
